\title{
Effect of 7 and 13-Valent Pneumococcal Conjugate Vaccines Different Number of Doses for Pneumonia Control in 2008 and 2010 Birth Cohort Children
}

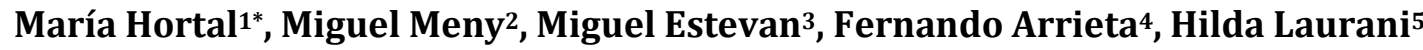 \\ ${ }^{1}$ Basic Sciences Program, Montevideo, Uruguay \\ ${ }^{2}$ Statistics Department, Montevideo City Council, Montevideo, Uruguay \\ ${ }^{3}$ Radiology Unit, Children's Hospital, Montevideo, Uruguay \\ ${ }^{4}$ Vaccination Department, Honorary Committee against Tuberculosis (CHLA), Montevideo, Uruguay \\ ${ }^{5}$ Honorary Committee against Tuberculosis (CHLA), Montevideo, Uruguay \\ Email: marujahortal@gmail.com
}

Received 31 December 2014; accepted 17 January 2015; published 20 January 2015

Copyright (C) 2015 by authors and Scientific Research Publishing Inc.

This work is licensed under the Creative Commons Attribution International License (CC BY).

http://creativecommons.org/licenses/by/4.0/

(c) (i) Open Access

\section{Abstract}

The 7-valent pneumococcal conjugate vaccine (PCV) was introduced in Uruguay in March 2008. In April 2010, it was replaced by PCV13. Surveillance of both vaccines was performed on hospitalized children with consolidated pneumonia. The effect of different number of vaccine doses was evaluated in 2008 and 2010 birth cohorts vaccinated with PCV7 and PCV13 respectively. The study aims to estimate the effects of PCV7 and PCV13 different number of doses on consolidated pneumonia, through the study of hospitalized children from 2008 and 2010 birth cohorts. Vaccination records of every child were available providing precise vaccination data; therefore a new approach was used to estimate PCVs effect. Incidence rate was calculated for each year of the study and for the different number of vaccine doses used each year. Exposure was calculated as person per year and rate ratio values determined the decrease of consolidated pneumonias. This decrease in percentage was estimated as the difference between the incidence with no vaccine and the incidence of every one of the doses. Incidence rate ratio revealed significant values for the three vaccine doses of PCVs for both cohorts. Upon comparing incidences, significant reduction percentages of consolidated pneumonia admissions were found. The reduction percentage of consolidated pneumonia for fully vaccinated (3 doses) patients was $69.3 \%$ and $84.6 \%$ for PCV7 and PCV13, respectively. These results confirm that PCV7 and PCV13 are highly effective for reducing pediatric hospitalizations due to consolidated pneumonia, as reported by other national publications and demonstrated by international researchers.

\footnotetext{
${ }^{*}$ Corresponding author.
}

How to cite this paper: Hortal, M., Meny, M., Estevan, M., Arrieta, F. and Laurani, H. (2015) Effect of 7 and 13-Valent Pneumococcal Conjugate Vaccines Different Number of Doses for Pneumonia Control in 2008 and 2010 Birth Cohort Children. World Journal of Vaccines, 5, 37-42. http://dx.doi.org/10.4236/wjv.2015.51005 
Keywords

Pneumococcal Conjugate Vaccine, Pneumonia, Birth Cohort, Incidence Rate, Dose Schedule

\section{Introduction}

Pneumococcal invasive infections are causes of hospitalizations worldwide, including bacteremia, pneumonia and meningitis. Also in Uruguay the burden of these pneumococcal diseases urged control measures to prevent severe morbidity and mortality among children.

Because that reason, the heptavalent pneumococcal conjugate vaccine (PCV) was introduced in Uruguay in March 2008. It was administered in a 3-dose schedule (at 2, 4 and 12 months of age) to the cohort born in the same year. Universal vaccination with PCV7 was in use until March 2010, when it was replaced by PCV13which had an expanded formulation with six additional serotypes. Children who had started the vaccination schedule with PCV7 completed it with PCV13. Since April 2010 the cohort born that year was exclusively vaccinated with PCV13.

Epidemiological consequences of a National Immunization Program (NIP) differ from the results of field trials. Therefore, it is mandatory to evaluate the effect of vaccination on the general population by assessing the incidence of consolidated pneumonias [1]. However, information on the impact of both pneumococcal conjugate vaccines is scarce in Uruguay today. A four year population-based surveillance (2009-2013) of hospitalized children with pneumonia was carried out in a selected area of the northwest of Uruguay [2]. It was performed while the NIP was in progress, so it provided data on the vaccination status of in-patients with consolidated pneumonia. Also, it is worth mentioning that the NIP keeps individual immunization records of all vaccinated children born in the country [3]. Thus, with the information available on the vaccination histories, this study aims to estimate the effects of PCV7 and PCV13 vaccine different number of doses on consolidated pneumonia in hospitalized children, considering 2008 and 2010 birth cohorts.

\section{Methods}

\subsection{Population Surveillance}

Population-based surveillance for pneumonia in hospitalized children was carried out in a selected area of the northwest of the country with 238002 inhabitants (2011 National Census), 25.4\% of which represented the pediatric population. Surveillance started on January $1^{\text {st }}$, 2009, ten months later the implementation of PCV7 (Prevenar, Wyeth Pharmaceuticals Inc.) began. In March, 2010, it was replaced by PCV13 (Prevenar, Wyeth Pharmaceuticals Inc, markered by Pfizer Inc.) and the surveillance was in progress until March $31^{\text {st }}$, 2013. Patients were referred to four hospital ( 2 public and 2 private) located in the two most important urban centers into the selected study area.

The same methodology has been employed in studies pre- and post- the introduction of PCV, the results of which have been already published [1] [2]. Briefly, all hospitalized children with an acute lower respiratory tract infection were eligible if an X-ray was ordered to confirm pneumonia. Chest radiographies were digitized and informed blind for clinical diagnosis and vaccination status. Images were interpreted by a reference radiologist, following WHO guidelines [4]. Demographic and selected clinical data were abstracted into an abbreviated form, including PCV information (date of doses) documented by an official immunization certificate.

\subsection{Study Population}

Present study analyzed two birth cohorts. One of them comprised 3993 children born in 2008 and other one 3, 822 children born in 2010, vaccinated with PCV7 and PCV13 respectively. Males predominated in both cohorts, (51.6\% and 52.1\%). The 2008 cohort was followed during 2009, when the age of children averaged 18.2 months. The 2010 cohort was followed from January $1^{\text {st }}$, 2010 to March 31 $1^{\text {st }}$, 2013. Analysis was performed by year: age of children averaged 18.2 months in 2011 and 30.2 months in 2012.

A unique NIP register system provided information on the status of vaccination for all children born in Uruguay. Individual records included demographic data and all the NIP vaccines (type of vaccine, number and date 
of doses). Therefore the vaccination history of the children belonging to 2008 and 2010 birth cohorts was available, providing data on time of exposure before different PCVs doses were administered.

\subsection{Definitions}

Case: hospitalized patient with consolidated pneumonia X-ray documented

Non-case: all children in the birth cohorts, but some children are non-case until hospitalized due to a consolidated pneumonia.

Vaccinated: with 1, 2 or 3 vaccine doses after 20 days of being vaccinated

Pneumonia end-point: was consolidated pneumonia. WHO definition proposed alveolar consolidation or pleural effusion as an epidemiological X-ray marker.

Exposed: non-vaccinated, but time is recorded once the child is two months old.

Exclusion criteria: 1) Patient hospitalized without X-ray confirmed pneumonia; 2) hospital-acquired pneumonia; 3) children no longer residing in the selected study area.

Ethics The study protocol was approved by the National Ethics Committee of the Ministry of Health (ref. No 001-3-5929/2010) and endorsed by the Minister of Health.

No informed consent was required by the Committee for this observational study.

\subsection{Analysis}

The analysis of the data included in this manuscript aimed to demonstrate the effect of different number of PCVs doses on the two birth cohorts using different statistic procedures.

Incidence rate was estimated by calculating the number of patients hospitalized due to consolidated pneumonia (numerator) and the time of exposure of all children until they were vaccinated or hospitalized due to consolidated pneumonia (denominator).

The analysis was performed for each observed year and for the different number of vaccine doses per years and exposure to consolidated pneumonia was calculated as $10^{5}$ person-year.

Point estimation of ratio measures of effect involved taking the ratio of the observed values of incidence. The point estimate of incidence rate ratio was calculated for both birth cohorts and their respective number of doses, dividing the incidence rate of the exposed children by the incidence rate of the non-exposed [5] [6].

For the approximate interval estimation of rate ratio a logarithmic transformation was used to compensate for the asymmetric sampling distribution [7].

The percentage of the decrease of incidence was estimated as the difference between the incidence of no vaccine and the incidence of every one of the doses.

Another presentation of results in percentages was calculated as the difference between the incidence of non-vaccinated (expected cases) minus the incidence of each vaccine dose (observed cases) divided by the incidence of non vaccinated.

\section{Results}

In March 2008, PCV7 was introduced for children born that year, but the cohort was observed since January $1^{\text {st }}$, 2009, covering 73 hospitalized cases with consolidated pneumonia and analyzing the pneumococcal vaccination status for the entire cohort. Data on individual vaccination status enabled incidence rate calculation, a new approach involving the effect of PCV7 different doses on consolidated pneumonia hospitalization. Table 1 shows significant incidence rate ratio positive values for PCV7 three doses resulting from incidence relations of exposed and non-exposed cohort members.

Difference between incidences showed relevant reduction percentages. Confirming the effect of PCV7, high percentages of reduction for the different vaccine doses were determined: $57.7 \%, 66.2 \%$ and $69.3 \%$ for $1^{\text {st }}, 2^{\text {nd }}$ and $3^{\text {rd }}$ doses respectively (Table 2).

PCV13 replaced PCV7 in March, 2010. Since April it was exclusively used, starting by the 2010 birth cohort, which was followed until March $31^{\text {st }}$, 2013. Ninety seven out of the 3822 children in the cohort were cases hospitalized due to consolidated pneumonia. Table 3 shows the evaluation of the incidence of exposed children versus non-exposed children, which revealed significant incidence rate ratios for the different PCV13 doses.

The 2010 birth cohort surveillance (2010-2012) also revealed high reduction percentages (Table 4) For the 
first dose the reduction percentage was $68.9 \%$, for the $2^{\text {nd }}$ dose it was $78.9 \%$ and $84.7 \%$ after the booster dose. Similar percentages were obtained when the expected cases were analyzed vs the observed cases. Figure 1 shows graphically the dramatic reduction on the observed cases according the number of administered vaccine doses.

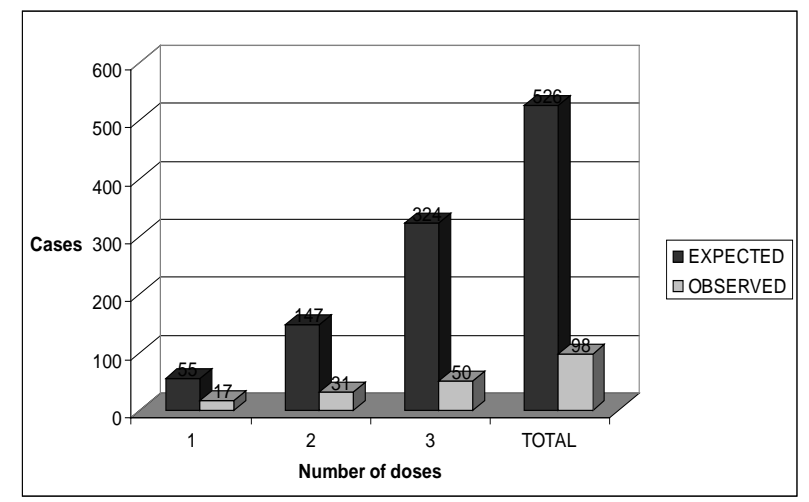

Figure 1. Number of expected cases without vaccine and the reduction of the number of observed cases according the different number of administered vaccine doses.

Table 1. Birth cohort 2008 observed during year 2009.

\begin{tabular}{cccccc}
\hline Vaccine & Cases & Person year & Incidence rate $^{\mathrm{a}}$ & Incidence rate ratio $^{\mathrm{b}}$ & $\mathrm{CI}^{\mathrm{c}}$ \\
\hline No vaccine & 12 & 209 & 5742 & & \\
1 dose & 16 & 629 & 2542 & 2.3 & $1.1-4.8$ \\
2 doses & 36 & 1858 & 1938 & 3.0 & $1.5-5.7$ \\
3 doses & 21 & 1191 & 1763 & 3.3 & $1.6-6.6$ \\
Total vaccinated & 73 & 3679 & 1984 & 2.9 & $1.6-5.3$ \\
\hline
\end{tabular}

a. Per 100,000. b. Non-vaccinated vs. vaccinated. c. Confidence intervals of rate ratio.

Table 2. Effect of PCV7 different doses for reduction of 2008 cohort patients hospitalizations due to consolidated pneumonias.

\begin{tabular}{ccccc}
\hline Vaccine & Cases & Person year & Incidence & \% Reduction \\
\hline No vaccine & 12 & 209 & 5742 & 55.7 \\
1 dose & 16 & 629 & 2542 & 66.2 \\
2 doses & 36 & 1858 & 1938 & 69.3 \\
3 doses & 21 & 1191 & 1763 & \\
\hline
\end{tabular}

Table 3. Birth cohort 2010 observed April 2010 through March 2013.

\begin{tabular}{cccccc}
\hline Vaccine & Cases & Person year & Incidence rate $^{\mathrm{a}}$ & Incidence rate ratio $^{\mathrm{b}}$ & $\mathrm{CI}^{\mathrm{c}}$ \\
\hline No vaccine & 9 & 158 & 5679 & & $1.4-7.2$ \\
1 dose & 17 & 961 & 1768 & 3.2 & $2.3-9.9$ \\
2 doses & 31 & 2583 & 1200 & 4.7 & $3.2-13.2$ \\
3 doses & 50 & 5714 & 875 & 6.5 & $2.7-10.7$ \\
Total vaccinated & 97 & 9258 & 1048 & 5.4 & \\
\hline
\end{tabular}

a. Per 100,000. b. Non-vaccinated vs. vaccinated. c. Confidence intervals of rate ratio. 
Table 4. Effect of PCV13 different doses for reduction of 2010 cohort patients hospitalizations due to consolidated pneumonia.

\begin{tabular}{ccccc}
\hline Vaccine & Cases & Person year & Incidence & \% Reduction \\
\hline No vaccine & 9 & 158 & 5679 & 68.9 \\
1 dose & 17 & 961 & 1768 & 78.9 \\
2 doses & 31 & 2583 & 1200 & 875.6 \\
3 doses & 50 & 5714 & 875 & 84.6 \\
\hline
\end{tabular}

\section{Discussion}

To the best of our knowledge, this is the first study which shows the effect of different PCVs doses on consolidated pneumonia in hospitalized birth cohort children. The study revealed the effect of PCV7 and PCV13 with 2 +1 schedule in two birth cohorts [8]. Significant incidence rate ratio values were found for hospitalized children with consolidated pneumonia, in a certain area of Uruguay. Also, high percentages of reduction for the different PCVs doses were demonstrated. The success of these vaccines is also due to the high vaccine compliance. Observational studies have limitations for establishing cause and effect, but the decline in the incidence of pneumonia in Uruguay and in other countries where vaccination has been implemented supports these results. Besides, a 56\% reduction in the consolidated pneumonia hospitalization rates pre and post PCV7 confirmed the effect of this vaccine [9].

Although incidence rate estimation is infrequent for assessing vaccine effect, it is appropriate when dealing with a dynamic population as our birth cohorts. The existence of an extraordinary register for the individual vaccination history of all children born in Uruguay provided data on time of exposure before the administration of PCVs.

Incidence rate ratio values increased when the number of doses progressed confirming the effect of PCV7 and PCV13. Another approach showed high percentages of exposure reduction (protection) for PCV7 and PCV13. The percentages of reduction achieved by the first doses of both vaccines were worth mentioning. Thus a national study of the effectiveness of PCV7 for the control of invasive infections was $82.7 \%$ for the first dose. The same study indicated the effectiveness of PCV7 was 94.8\% for 2 or more doses [10] [11].

The PCV7 and PCV13 booster doses of our study showed reductions of $69.3 \%$ and $84.7 \%$ respectively. Another study revealed a significant decline of $72 \%$ for lobar pneumonia admissions in Canadian children [12]. A population-based study in Israel demonstrated the effectiveness of PCVs was $40.7 \%$, for the control of alveolar pneumonias [13]. However two Italian studies that evaluated the impact of $2+1$ schedule found significant reductions for pneumonia endpoints [14] [15].

Our results are limited by the small number of cases recorded, although confidence intervals support our data. Patients with no vaccine might belong to a disadvantaged group of people. Because of that reason, we examined the characteristics of non-vaccinated (dwelling area, health care public/private, vaccination history) and found they did not differ from the vaccinated cases, even if they could have been considered as in a disadvantageous situation.

The two cohorts cannot be compared and this is a further limitation. Observation time in each cohort was different. Despite the limitations of observational investigation data, our results represent a new approach for the evaluation of the effects of PCVs either global or per individual doses for the reduction of hospital admissions of children with consolidated pneumonia. Studies on larger birth cohorts would provide robust statistic information to validate the presented results.

\section{Acknowledgements}

We are grateful for the valuable clinical data contribution of E Arreseigor, MD, L Martinez Arroyo, MD, R Herrmann, MD, and H Doti, MD. To the field work nurses. G Barrios and M Alves, To J Leiva, MD, and F Morales from the Archive Dept. and to S Juanicotena for her technical contribution. Also to all the NIP records personnel. 


\section{References}

[1] Hortal, M., Estevan, M., Laurani, H., Iraola, I. and Meny, M., Paysandú/Salto Study Group (2012) Hospitalized Children with Pneumonia in Uruguay: Pre and Post Introduction of 7 and 13-Valent Pneumococcal Conjugated Vaccines into the National Immunization Program. Vaccine, 33, 4934-4938. http://dx.doi.org/10.1016/j.vaccine.2012.05.054

[2] Hortal, M., Estevan, M., Meny, M., Iraola, I. and Laurani, H. (2014) Impact of Pneumococcal Conjugate Vaccine on the Incidence of Pneumonia in Hospitalized Children after Five Years of Its Introduction in Uruguay. PLoS One, 9, e98567. www.plosone.org http://dx.doi.org/10.1371/journal.pone.0098567

[3] Ronveaux, O., Arrieta, F., Curto, S., Laurani, H. and Danovaro-Holliday, M.C. (2006) Assessment of the Quality of Immunization Data Produced by the National Individual Registration System in Uruguay, 2006. Revista Panamericana de Salud Pública, 26, 153-160.

[4] (2001) World Health Organization Pneumonia Vaccine Trial Investigator's Group, Standardization of Interpretation of Chest Radiographs for the Diagnosis of Pneumonia in Children, World Health Organization Geneva.

[5] Pita Fernández, S., Pértigas Díaz, S. and Valdés Cañedo, F. (2013) Medidas de frecuencia de enfermedad. Madrid: Fisterra, Disponible en. http://www.fisterra.com/mbe/investiga/medidas_frecuencia/med_frec2.pdf

[6] Prentice, R.L. (1986) A Case-Cohort Design for Epidemiologic Cohort Studies and Disease Prevention Trials. Biometrika, 73, 1-11. http://dx.doi.org/10.1093/biomet/73.1.1

[7] Rothman, K.J. (1986) Modern Epidemiology. 5. Measures of Disease Frequency, 23-34. Little, Brown and Company. Boston/Toronto.

[8] O’Brien, K.K., Goldblatt, D. and Witney, C.G. (2014). Why Do We Need a Systematic Review of Pneumococcal Conjugate Vaccine Dosing Schedules? Pediatric Infectious Disease Journal, 33, S107-S108. http://dx.doi.org/10.1097/INF.0000000000000075

[9] Pirez, M.C., Algorta, G., Sobrero, H., Varela, A., Giachetto, G. and Montano, A. (2011) Impact of Pneumococcal Universal Vaccination of Hospitalizations for Pneumonia and Meningitis in Children in Montevideo, Uruguay. Pediatric Infectious Disease Journal, 30, 669-674. http://dx.doi.org/10.1097/INF.0b013e3182152bf1

[10] Picon, T., Alonso, L., Garcia Garrabot, G., Speranza, N., Casas, M., Arrieta, F., Camou, T., Rosa, R., De Oliveira, L.H., Verani, J.R. (2013) Effectiveness of the 7-Valent Pneumococcal Conjugate Vaccine against Vaccine-Type Invasive Disease among Children in Uruguay: An Evaluation Using the Existing Data. Vaccine, 315, C105-C113.

[11] Huebner, R.E., Mbelle, N., Forrest, B., Madore, D.V. and Klugman, K.P. (2002) Immunogenicity after One, Two or Three Doses and Impact on the Antibody Response to Coadministered Antigens of a Nonavalent Pneumococcal Conjugate Vaccine in Infants of Sweto, South Africa. Pediatric Infectious Disease Journal, 21, 1004-1007. http://dx.doi.org/10.1097/00006454-200211000-00006

[12] De Wals, P., Robin, E., Fortin, E., Thibeault, R., Ouakki, M. and Douville-Frader, M. (2008) Pneumonia after Implementation of Pneumococcal Conjugate Vaccine Program in the Province of Quebec, Canada. Pediatric Infectious Disease Journal, 27, 963-968. http://dx.doi.org/10.1097/INF.0b013e31817cf76f

[13] Verani, J.R., Givon-Lavi, R., Whitney, C.G., Greenberg, D. and Dagan, R. (2014) Effectiveness of Pneumococcal Conjugate Vaccine against Pneumonia in Israel. Pneumonia, S3, 278.

[14] Esposito, S., Lizioli, A., Lastrico, A., Begliatti, E., Rognoni, A., Tagliabue, C., Cesari, L., Carreri, V. and Principi, N. (2007) Impact on Respiratory Tract Infections If Heptavalent Pneumococcal Conjugate Vaccine Administred at 3, 5 and 11 Months of Age. Respiratory Research, 8, 12. http://www.ncbi.nlm.nih.gov/pmc/articles/PMC1804265/

[15] Ansaldi, E., Sticchi, L., Durando, P., Begliatti, E., Rognoni, A., Tagliabue, C., Crovari, P. and Icardi, G. (2008) Decline in Pneumonia and Acute Otitis Media after the Introduction of Childhood Pneumococcal Vaccination in Liguria, Italy. Journal of International Medical Research, 26, 1255-1260. http://dx.doi.org/10.1177/147323000803600612 
Scientific Research Publishing (SCIRP) is one of the largest Open Access journal publishers. It is currently publishing more than 200 open access, online, peer-reviewed journals covering a wide range of academic disciplines. SCIRP serves the worldwide academic communities and contributes to the progress and application of science with its publication.

Other selected journals from SCIRP are listed as below. Submit your manuscript to us via either submit@scirp.org or Online Submission Portal.
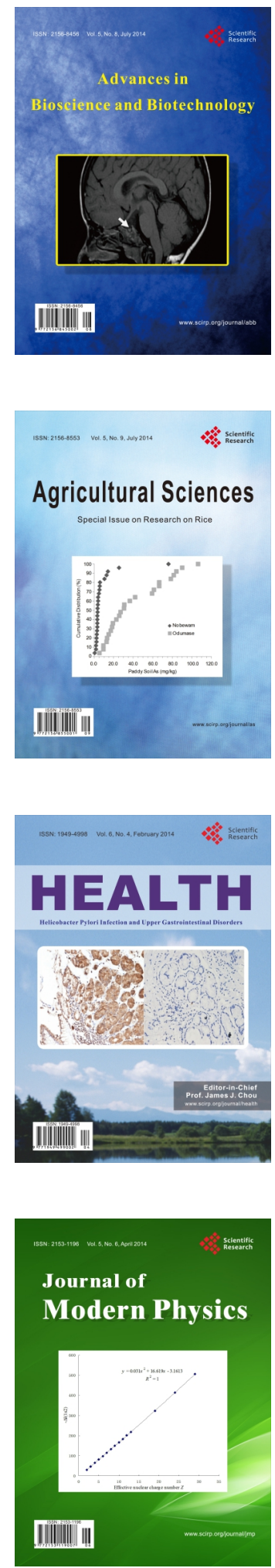
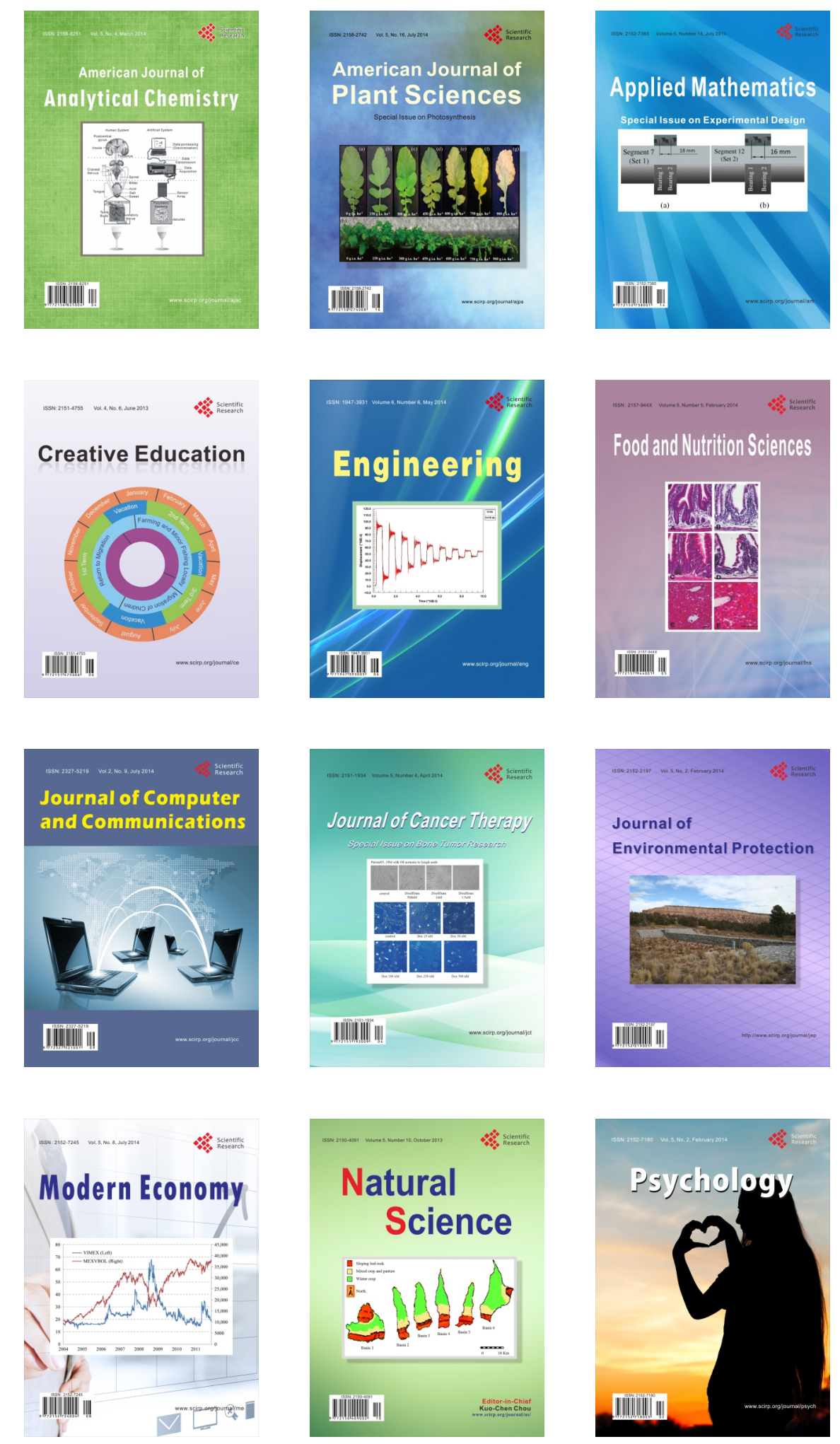